\title{
Construction and tests of the research stand with built-in correctors for the detection of gas outflow from long pipelines
}

\author{
Karol Golak ${ }^{1 *}$, Pawet Lindstedt ${ }^{2}$, Błażej Bartoszewicz ${ }^{1}$ \\ ${ }^{1}$ Faculty of Mechanical Engineering, Bialystok University of Technology, Wiejska 45C street, 15-351 \\ Białystok, Poland \\ ${ }^{2}$ Air Force Institute of Technology, Księcia Bolesława 6 street, 01-494 Warszawa, Poland
}

\begin{abstract}
The localization and identification of small gas leaks from a damaged gas pipeline is a very important but at the same time problematic and difficult process to carry out. Quick identification, estimation and precise localization of the leak leads to minimizing the financial and equipment losses resulting from damage. The most commonly used continuous internal methods of leak identification are based on pressure and mass intensity measurements flow at various places in the pipeline. The article presents a description of the construction of a test stand characterized by the correctors built in it and the examples of the signals obtained from its tests for different values of simulated leaks. The results obtained will be used to develop a new method for identifying and locating leakages from long pipelines. This method is based on the cross power spectral density of measured pipeline standard signals (pressure and mass flow) and the membrane displacement signals delivered from the test equipment (correctors) connected to the system.
\end{abstract}

\section{Introduction}

The gas pipelines are used for decades and during this period, due to progressive corrosion, mechanical leaks may occur. Gas leaks from long pipelines can cause serious accidents and may damage the equipment, hence it is important to detect, estimate and locate them as soon as possible.

There are a number of methods to detect the occurrence of a gas leak, generally they can be divided into non-continuous (e.g. smart pigging, helicopter inspection) and continuous monitoring. $[1,2]$

Non-continuous methods do not allow for constant observation and assessment of the pipeline's technical condition. Individual inspections are carried out at certain period of time which often does not allow for instant leak detection. [4, 7]

Continuous methods are used without interruption during the entire operation of the pipeline. They could be divided into external and internal based systems. [2, 3, 6] Externally based systems detect leaking product outside the pipeline (acoustic systems, fiber optic, dielectric cables, laser sensors). Internal based systems (e.g. pressure analysis, mass balance method), known as computational pipeline monitoring (CPM) uses different types of sensors to monitor internal pipeline parameters which are a basis for different methods of gas leak detection, however, in order to use them, it is necessary to use them in order to use them. installation of leak detection systems before the pipeline is started. [9, $11,12,13,14]$

The article presents the construction and test results of a test stand for simulating gas leaks from long pipelines. The stand is characterized by two correctors attached to the

${ }^{\star}$ Corresponding author: k.golak@pb.edu.pl 
pipeline which provide an additional diagnostic signal in the form of weak interaction signals. $[5,8,10]$ The connection of correctors to the pipelines does not affect the quality of the pipeline and is easy to implement.

\section{Construction of a test stand with built-in correctors to detect gas leaks from the pipeline}

\subsection{Construction of a test stand}

Test stand built on the basis of the scheme in Fig.1. is shown in Fig. 2. The stand was built with about 27 meters of welded PPR pipes with an inner diameter of $\varnothing 45 \mathrm{~mm}$ mounted on a frame of aluminium structural profiles. At the beginning of the pipeline there is an air tank (V) with a capacity of $0,025 \mathrm{~m}^{3}$, to which atmospheric air is pumped using a compressor. Between the compressor and tank there is a compressed air preparation unit for adjusting pressure to the required level of working and for cleaning the. Along with the tank, the entire system has a capacity of about $0,075 \mathrm{~m}^{3}$.

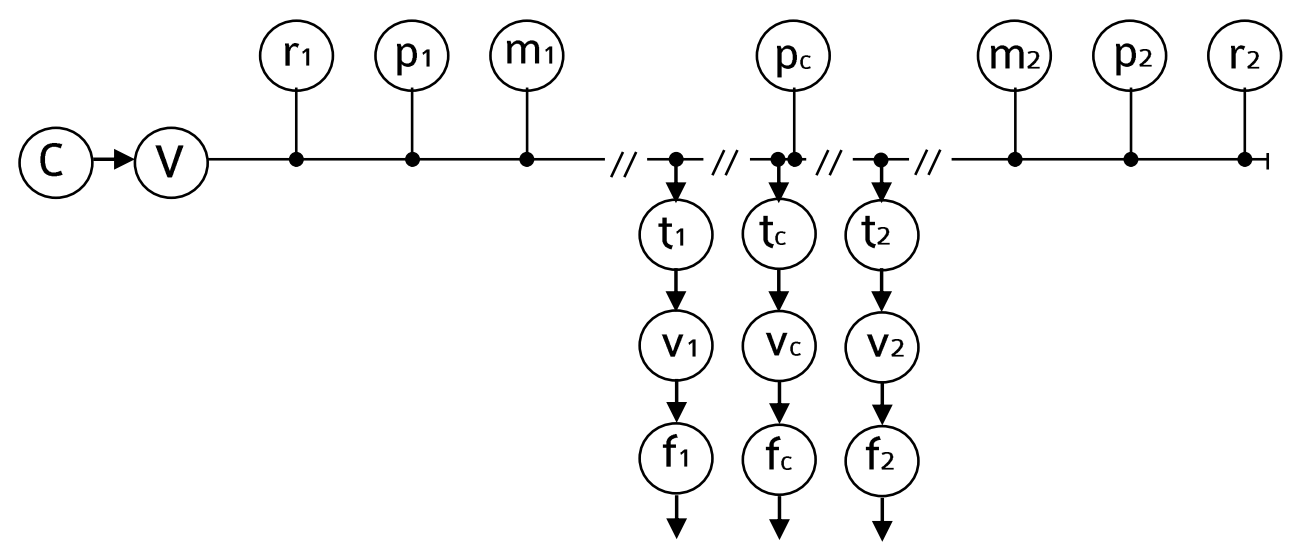

Fig. 1. Scheme of the test stand for leakage simulations from pipelines, where: $C$ - compressor, $V$-pressure tank, $r$ - correctors, $p$ - pressure sensors, $m$ - mass flow sensors, $t$ - taps, $\mathrm{v}$ - adjustable valves, f-flow meters

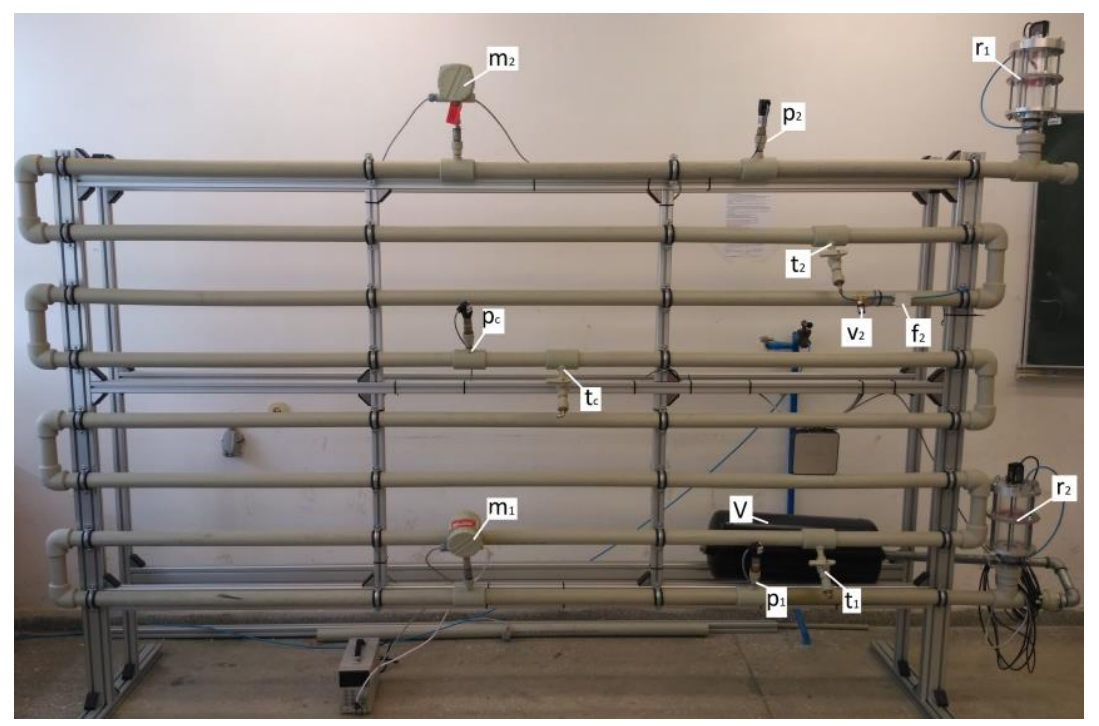

Fig. 2. Test stand for leakage simulations from pipelines with mounted correctors. 
Behind the tank and at the end of the system are mounted correctors $\left(r_{1}, r_{2}\right)$. Pressure sensors $\left(p_{1}, p_{c}, p_{2}\right)$ and mass flow sensors $\left(m_{1}, m_{2}\right)$ have been installed in the pipeline in the order shown in Fig. 1. The taps at the beginning $\left(t_{1}\right)$, centre $\left(t_{c}\right)$ and end of the pipeline $\left(t_{2}\right)$ are used to simulate gas leaks. In order to enable precise control and measurement of the amount of leakage behind the tap from which the leak is simulated, a solenoid valve and a flow meter are installed.

Data instruments from National Instruments were used to collect data and control the simulated leaks volume.

\subsection{Construction of the correctors}

At the beginning and the end of the test stand, two additional devices - correctors $\left(r_{1}\right.$ and $\left.r_{2}\right)$ were built using the tees. The corrector was built in the form of two chambers separated from each other by a rubber membrane with a mass installed in the middle (brass sheet). The membrane and its mass have been selected in such a way that there is visible continuous resonance vibrations. The chambers are connected with each other by a plastic pipe with an internal diameter of $\varnothing 4 \mathrm{~mm}$, which allows pressure equalization between two correctors parts. On the membrane with the mass mounted on its centre, as a result of the gas leak its vibrations will be visible. A high-precision laser distance sensor was used to measure the vibrations of the membrane. The measurement is carried out by glass installed on the top of the corrector which transmitting the light with wavelength emitted by the sensor.

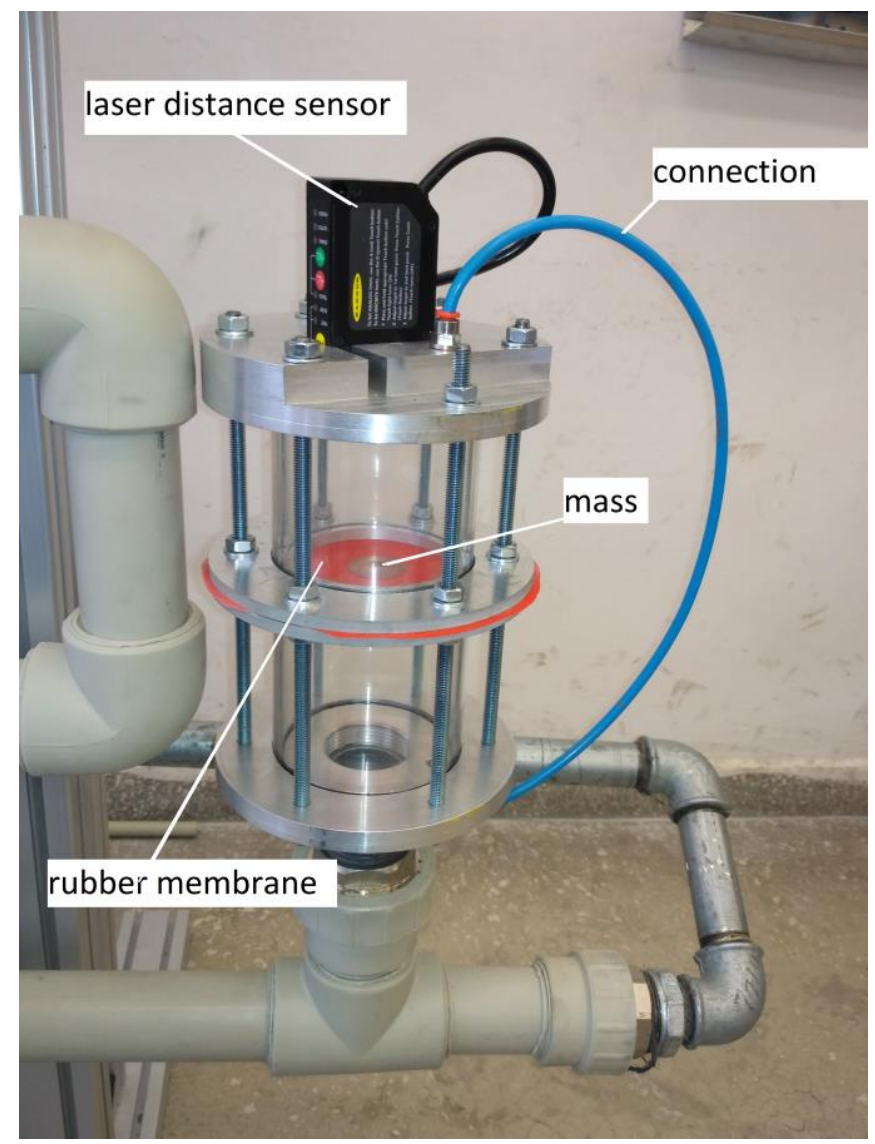

Fig. 3. Corrector build-in test stand. 
The correctors built into the test stand are to provide an additional diagnostic signal - it is a device that transforms the pressure change that appears in the pipeline as a result of a leakage on the membrane displacement signal.

\section{The test results of the test stand}

The test stand tests were carried out at the pressure of about $0.6 \mathrm{MPa}$. The leakage control solenoid was set to obtain different leakages. The research was carried out by simulating leakages from three places in the pipeline $\left(t_{l}, t_{c}\right.$ and $\left.t_{2}\right)$. Each measurement lasted 6.5 seconds from the moment of the leak occurrence and during this time the following data was recorded with indicators describing their position in the pipeline 1 for the beginning, $c$ - centre, 2 end of the pipeline:

- $p_{1}, p_{c}, p_{2}$ - pressure in the pipeline $[\mathrm{Pa}]$

- $r_{1}, r_{2}$ - membrane displacement [mm].

Leak volumes:

- $f 10$ - 10 litres per minute $\left(0.000166 \mathrm{~m}_{3} / \mathrm{s}\right)$;

- $f 20$ - 20 litres per minute $\left(0.000333 \mathrm{~m}_{3} / \mathrm{s}\right)$;

- $f 30$ - 30 litres per minute $\left(0.000499 \mathrm{~m}_{3} / \mathrm{s}\right)$;

- $f 40$ - 40 litres per minute $\left(0.000666 \mathrm{~m}^{3} / \mathrm{s}\right)$;

- $f 50$ - 50 litres per minute $\left(0.000833 \mathrm{~m}^{3} / \mathrm{s}\right)$;

The sample results for the different gas leakage volume simulation from $t_{2}$ (valve on the end of stand) is presented from Fig. 4 to Fig. 8

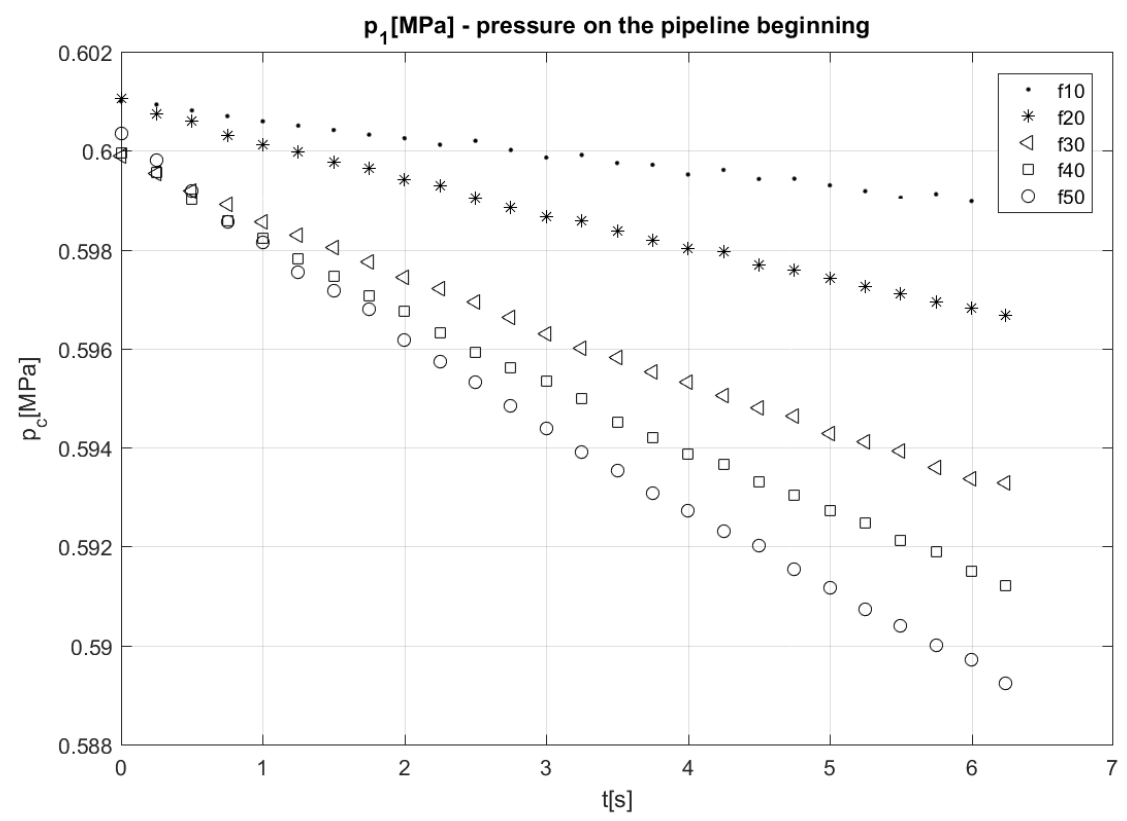

Fig. 4. Pressure $p_{I}[\mathrm{MPa}]$ signal waveform as a result of leakage simulation by a tap $t_{2}$. 


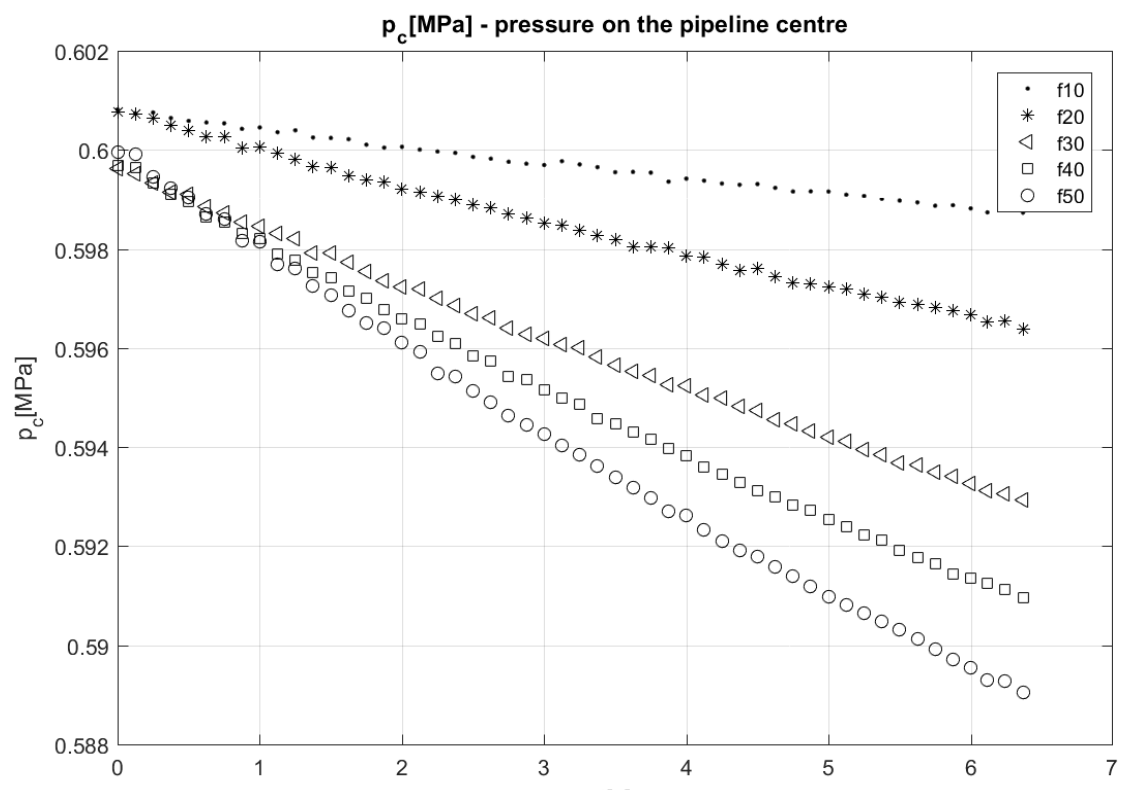

$\mathrm{t}[\mathrm{s}]$

Fig. 5. Pressure $p_{c}[\mathrm{MPa}]$ signal waveform as a result of leakage simulation by a tap $t_{2}$.

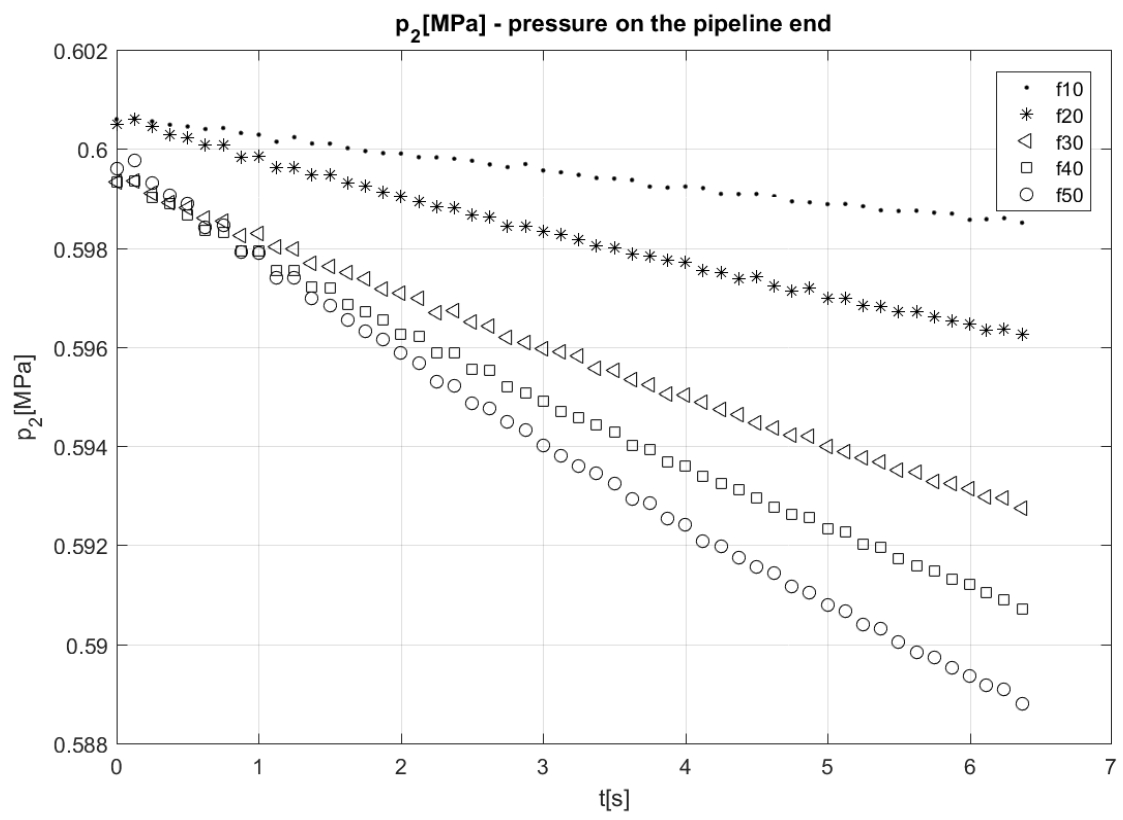

Fig. 6. Pressure $p_{2}[\mathrm{MPa}]$ signal waveform as a result of leakage simulation by a tap $t_{2}$. 


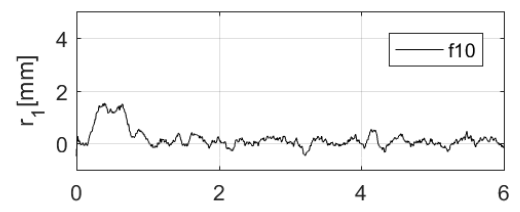

$\mathrm{t}[\mathrm{s}]$
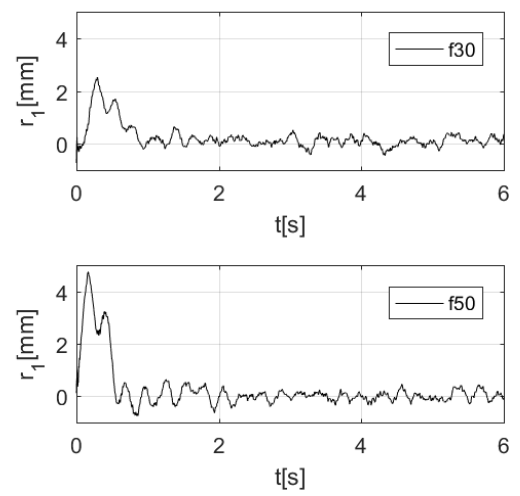

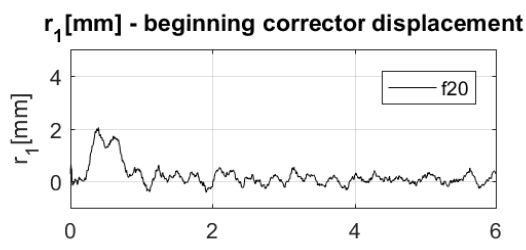

$\mathrm{t}[\mathrm{s}]$

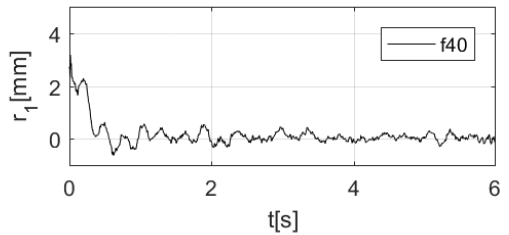

$\mathrm{t}[\mathrm{s}]$

Fig. 7. Membrane displacement in corrector $r_{l}[\mathrm{~mm}]$ as a result of leakage simulation by a tap $t_{2}$.

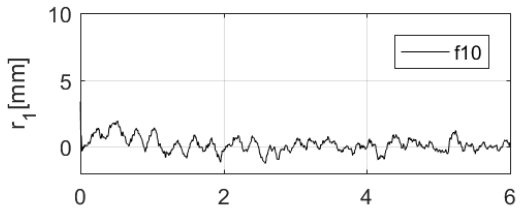

$\mathrm{t}[\mathrm{s}]$

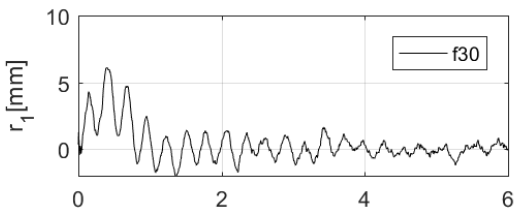

$\mathrm{t}[\mathrm{s}]$

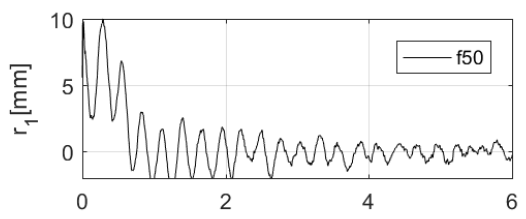

$\mathrm{t}[\mathrm{s}]$

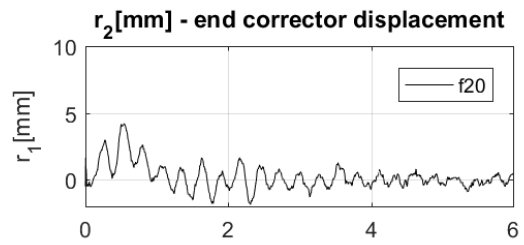

$\mathrm{t}[\mathrm{s}]$

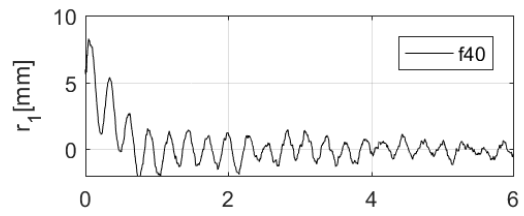

$\mathrm{t}[\mathrm{s}]$

Fig. 8. Membrane displacement in corrector $r_{2}[\mathrm{~mm}]$ as a result of leakage simulation by a tap $t_{2}$.

From the Fig. 4. to Fig. 8. the results of measurements with simulated small leakages are presented. This results confirm that correctors connected to the pipelines, can provide the new valuable diagnostic information that could increase the effectiveness of leaks identification, estimation and localization. 


\section{Summary}

The article presents a research stand for simulating the leakages of gas from long gas pipelines characterized by additional two research equipment - correctors placed at the inlet and outlet of the pipeline and standard mounted pressure sensors and mass flow sensors.

Attaching correctors to the gas pipeline have a purpose to obtain additional valuable diagnostic information, which are weak interactions signals. Unlike standard pressure signals, the signals of weak interactions are more sensitive and more resistant to noise. The use of weak interaction signals can complement existing mass flow and pressure signals and can be used to identify the location and size of gas outflow.

Signals from the system and research devices (correctors) will be the basis for the development of a new method for testing the outflow from the gas pipeline based on the quotient of the spectral power density of signals generated by the corrector and measured signals of pressure and mass flow.

Karol Golak part of paper was supported by the research project No. S/WM/2/2017 and financed by the Ministry of Science and Higher Education.

Rafat Gradzki part of paper was supported by the research project No. S/WM/1/2016 and financed by the Ministry of Science and Higher Education.

\section{References}

1. L. Boaz, S. Kaijage, R. Sinde, An overview of pipeline leak detection and location systems. In: Science, Computing and Telecommunications (PACT), 2014 Pan African Conference on. IEEE;133-137 (2014).

2. API 1130, Computational pipeline monitoring (2nd ed.), American Petroleum Institute, Washington, DC, USA (2002)

3. W. Liang, L. Zhang, A wave change analysis (WCA) method for pipeline leak detection using Gaussian mixture model, Journal of Loss Prevention in the Process Industries, 25, Issue 1, pp. 60-69, (2012)

4. L. Billman, R. Isermann, Leak detection methods for pipelines, Automatica, 23, no. 3, 381-385, (1987).

5. P. Ostapkowicz, Leakage detection from liquid transmission pipelines using improved pressure wave technique, Maintenance and Reliability, 16, no. 1, 9-16 (2014)

6. K. E. Abdulimen, A. A. Susu, Liquid pipeline leak detection system: model development and numerical simulation, Chemical Engineering Journal, 97, Issue 1, (2004).

7. Z. Kowalczuk, K. Gunawickrama, Detecting and locating leaks in transmission pipelines, In J. Korbicz, J. Koś cielny, Z. Kowalczuk, W. Cholewa (Eds.): Fault diagnosis. Models, Artifical Intelligence, Applications, Chapter 21, 821-864 (2004)

8. P. Lindstedt, Weak interactions between objects in the signal-based and parametric diagnostics of transport-dedicated complex engineering systems, Aircraft Engineering and Aerospace Technology, 77, no. 3, 222-227 (2005).

9. A. E. Liu,. Overview: Pipeline Accounting and Leak Detection by Mass Balance, Theory and Hardware Implementation (2008)

10. R. Grą dzki, K. Golak, P. Lindstedt, B. Bartoszewicz, Reasons for the experimental research of gas outflows based on the signals of weak interactions between the tested model of the gas pipeline, and tested equalizer, Journal of Konbin, 2, 39-48, (2015)

11. R. Grą dzki, P. Lindstedt, Method of assessment of technical object aptitude in environment of exploitation and service conditions. Eksploatacja i Niezawodnosc Maintenance and Reliability, 1; 54-63 (2015), http://dx.doi.org/10.17531/ein.2015.1.8

12. R. Grą dzki, P. Lindstedt, K. Golak, Premises of evaluation of the technical object suitability with including the quality of its maintenance and operation, and their 
initial conditions. In: Safety and Reliability: Methodology and Applications Proceedings of the European Safety and Reliability Conference, chapter 44; 319 326 (2015)

13. S. L. Scott, M. A. Barrufet, Worldwide Assessment of Industry Leak Detection Capabilities for Single \& Multiphase Pipelines, Project Report Prepared for the Minerals Management Service, OTRC Library Number: 8/03A120, (2003).

14. Y. Sivathanu, Natural Gas Leak Detection in Pipelines. U.S. Department of Energy, National Energy Technology Laboratory, (2003).

15. Tomasik P. Zastosowania modeli dokładnych sieci do detekcji i lokalizacji wycieków. In: Diagnostics of Processes and Systems, 507-512, (2011). 